\title{
Hibridación in situ por fluorescencia (FISH) en muestras de líquido amniótico obtenido por amniocentesis precoz
}

\author{
Luz Mery Bernal Parra', Thomaz Rafael Gollop², Nadyr Naccache². \\ 1. Bióloga, Pontificia Universidad Católica Do Paraná. \\ Magister en Biología celular y Genética, Pontificia Universidad Javeriana. \\ Doctorado en Biología Genética, Universidad De Sao Paulo. \\ Docente Asistente, UNAD. \\ 2. Serviço de Ginecologia e Genética de São Paulo, Brasil
}

Correspondencia: luz.bernal@unad.edu.co

Recibido: 06-11-11/Aceptado: 11-11-11

\begin{abstract}
Resumen
La hibridación in situ por fluorescencia (FISH) se puede utilizar para diagnosticar prenatalmente las aneuploidías más frecuentes. Se utilizan núcleos interfásicos de células fetales no cultivadas. Este es un avance importante en el diagnóstico prenatal. Se describe la aplicación de la FISH en 15 muestras de líquido amniótico no cultivado extraído entre la $12 \pm 7.5$ y $146 / 7$ semanas de gestación ( $\mathrm{m} \pm 1$ sd $=$ $13,63 \pm 0,52)$. El volumen de líquido amniótico recolectado oscilo entre de 2 y $4 \mathrm{ml}(3,16 \pm 0,58)$. Todas las pacientes presentaban como principal indicación para la realización del procedimiento la edad materna avanzada. El tiempo para obtener los resultados varió de I a 5 días (2,13 \pm 1,55 días). El tiempo de entrega de los resultados citogenéticos osciló entre 13 y 22 días (1 8,4 \pm 3,2). En total, I 4 casos (93,33\%) fueron informativos. Las cinco aneuploidías diagnosticadas por FISH fueron confirmadas por citogenética tradicional, sin embargo, el análisis citogenético tradicional identificó un caso de rearreglo cromosómico equilibrado: [46, XY, t (16; 17) (q24; q I2) pat] y un caso de mosaicismo: 46,XX/47,XX, +mar. La realización de FISH requiere muchos pasos que pueden impedir que los resultados sean obtenidos, es importante obtener un número suficiente de núcleos para la hibridación. El estudio de aneuploidías en amniocitos no cultivados debe ser introducido en el protocolo de diagnóstico prenatal en mujeres embarazadas con riesgo aumentado de concebir un feto con aneuploidias.
\end{abstract}

Palabras clave: diagnóstico prenatal, la amniocentesis temprana, la interfase FISH.

\section{Abstract \\ Fluorescence in situ hybridization (FISH) in samples of amniotic fluid obtained by early amniocentesis}

Fluorescent in situ hybridization can be used to diagnose prenatally the most frequent aneuploidies. Nucleic from non-cultivated fetal cells are used. This is an important advance in prenatal diagnosis. We describe the application of FISH in I 5 non-cultivated amniotic fluid samples collected between $12 \pm 5 / 7$ and 14 $6 / 7$ weeks ( $m \pm$ I s.d. $=13.63 \pm 0,52$ ). Amniotic fluid volume ranged from 2 to $4 \mathrm{ml}(3,16 \pm 0.58)$. All indications were advanced maternal age. The time to obtain the results ranged from I to 5 days $(2.13 \pm$ I. 55 days). The times to deliver the cytogenetic results ranged from 13 to 22 days ( $18.4 \pm 3.2$ ). In total, 14 cases (93.33\%) were informative. The five aneuploidies diagnosed by FISH were confirmed after the 
traditional cytogenetic analysis. However, the cytogenetic analysis identified one balanced chromosomal rearrangement $[46, X Y, t(16 ; 17)(q 24 ; q / 2)$ pat $]$ and a case of mosaicism $46 . X X / 47 . X X$, + mar. Conducting FISH requires many steps that may prevent results from being obtained; the most important of these steps is to obtain an enough number of nucleic for hybridization. We conclude that the study of aneuploidies in non-cultivated amniocytes should be introduced to the prenatal diagnosis protocol in pregnant women with an increased risk of conceiving an aneuploid fetus.

Key words: prenatal diagnosis, early amniocentesis, interphase FISH.

\section{Resumo \\ Hibridação in situ por fluorescência (FISH) em amostras de liquido amniótico obtido por amniocentese precoce}

A incorporação da técnica de hibridação in situ por fluorescência (FISH) no diagnóstico das aneuploidias mais freqüentes utiliza núcleos interfásicos de células fetais não cultivadas. Trata-se de um importante avanço no diagnóstico pre-natal. Apresentamos a aplicação da FISH em I 5 amostras de líquido amniótico sem cultivar, coletado entre 12 5/7 a I4 6/7 semanas ( $m \pm$ I d.p. = 13,63 \pm 0,52). O volume de liquido amniótico variou de 2 a $4 \mathrm{ml}(3,16 \pm 0,58)$. Todas as pacientes apresentavam como principal indicação para a realização do procedimento a idade materna avançada. $O$ tempo para a obtenção dos resultados variou de I a 5 dias $(2,13 \pm 1,55)$. O tempo de entrega dos resultados citogenéticos variou de 13 a 22 dias ( $18,4 \pm 3.2)$. Do total, I 4 casos (93,33\%) foram informativos. As cinco aneuploidias diagnosticadas por FISH foram confirmadas pela citogenética tradicional. No entanto, a citogenética identificou um caso de rearranjo cromossômico equilibrado: $\{46, X Y, t($ I $;$; I )(q24;q I 2)pat $\}$ e um caso de mosaicismo: 46,XX/47,XX, +mar. A realização da FISH requer uma serie de etapas que podem afetar a obtenção de um resultado; a mais importante delas é a obtenção de uma quantidade adequada de núcleos para a hibridação. Consideramos que o estudo de aneuploidias em amniócitos não cultivados deve incorporar-se ao protocolo de diagnóstico pré-natal em gestantes com risco elevado para aneuploidia.

Palavras-chave: diagnóstico pré-natal, amniocentese precoce, FISH interfásico.

\section{Introducción}

El diagnóstico citogenético prenatal es ofrecido a mujeres embarazadas que presentan mayor riesgo de concebir un hijo con anomalía cromosómica. Después de la obtención de la muestra para análisis, un periodo de espera es necesario, lo que puede conducir a un significativo grado de ansiedad en las pacientes. La posibilidad de abreviar ese lapso, generalmente de 7 a 20 días, es de gran valía tanto para la paciente como para el médico. La hibridación in situ por fluorescencia (FISH) es una técnica capaz de detectar, en un corto plazo de tiempo (24-48h), las aneuploidías cromosómicas más frecuentes (13, $18,21, \mathrm{X}$ y $\mathrm{Y}$ ) a partir de núcleos interfásicos de células fetales no cultivadas $(1,2,3,4,5)$.

Numerosos trabajos sobre el uso de FISH en el diagnóstico prenatal fueron publicados. Aquellos con el mayor número de pacientes estudiadas son los de Klinger (1), Ward (6), D'Alton (7), Jalal (2) Eiben (3), Morris (4), Lewin (8), Thilaganathan (9) Chan-wei (10). Esta técnica ha adquiriendo cada vez más relevancia en el contexto del diagnóstico prenatal. Sin embargo, se trata de una técnica complementaria al análisis citogenético tradicional (11). En Brasil, la aplicación de la FISH en el diagnóstico prenatal es limitada por el alto costo y la difícil disponibilidad de sondas moleculares; por eso es aplicable a casos seleccionados. El objetivo de este estudio fue estandarizar en una institución brasilera de diagnóstico prenatal la técnica de FISH en núcleos interfásicos provenientes de líquido amniótico obtenido por amniocentesis precoz y comparar los resultados de este análisis con los de la citogenética tradicional. 


\section{Materiales y métodos}

La población de estudio consistió en embarazadas sometidas a amniocentesis precoz para la realización del diagnóstico citogenético prenatal en una institución brasilera de diagnóstico prenatal. En la consulta de consejería genética previa al procedimiento, se informaba que la FISH era un procedimiento en fase de implementación en la institución. Aunque los resultados de ese análisis estuvieran disponibles a la paciente, ella era advertida en cuanto a la necesidad de esperar por el diagnóstico definitivo. Este solo era concretizado con la emisión de un informe acerca del análisis citogenético tradicional. La colecta de líquido amniótico y el cultivo celular para el análisis citogenética tradicional fueron realizadas siguiendo la metodología de Naccache et al (10).

\section{Procesamiento del líquido amniótico para FISH}

Se utilizó una alícuota de 2 a $4 \mathrm{ml}$ del total de líquido amniótico recolectado por paciente. Solamente muestras translúcidas de coloración amarillenta fueron procesadas. Las muestras fueron centrifugadas por 10 minutos a $1.200 \mathrm{rpm}$. El sedimento fue resuspendido en $5 \mathrm{ml}$ de 1Xtripsina/ EDTA e incubado por 15 minutos a $37^{\circ} \mathrm{C}$. Después de centrifugación por 10 minutos a $1.200 \mathrm{rpm}$, el sedimento fue resuspendido en $5 \mathrm{ml}$ de solución hipotónica (ácido cítrico 1\% y Tween20 -10\%-). Las muestras fueron entonces incubadas durante 20 minutos a $37^{\circ} \mathrm{C}$. Después de centrifugación el sedimento fue fijado con metanol y ácido acético (3:1) y lavado por dos veces más. De dos a cuatro láminas por paciente (dependiendo del tamaño del sedimento) fueron preparadas. Una vez secas las preparaciones, fueron tratadas durante 10 minutos con una solución de formaldehido (1\%)/2XSSC. Se procedió a dos lavados en 2 XSSC durante 5 minutos y a un lavado en agua destilada durante 1 minuto. Las preparaciones secas a temperatura ambiente, fueron incubadas durante 2 minutos en una solución 2XSSC/Tween 20 (10\%).

\section{Sondas}

En la hibridación fueron utilizadas sondas de ADN para los cromosomas 13, 18, 21, X y Y, las cuales se encuentran comercialmente disponibles. Sondas específicas (Oncor) para las regiones centroméricas fueron utilizadas en la detección de los cromosomas sexuales-DXZ1 Xp11.1-q11.1 y DYZ3 Yp11.1-q11.1. En el caso del cromosoma 21, la sonda Oncor utilizada posee especificidad para el locus D21S55, localizado en 21q22.2. En algunos casos, fue utilizado el kit de la Vysis (AneuScreen) que evidencia simultáneamente los cromosomas $\mathrm{X}, \mathrm{Y}$ y 18 y o/13 y 21 . Se trata de una mezcla de sondas, cada una de las cuáles es complementaria a una región cromosómica con secuencia repetitivaDYZ3, DXZ1 y D18Z1 (18p11.1-q11.1). En el caso de los cromosomas 13 y 21, las sondas presentan secuencia única y son locus-específicas (LSI) para los cromosomas 13 (13q14, locus del gen del retinoblastoma) y 21 (21q22.13-q22.2).

\section{Hibridación in situ}

El protocolo de hibridación varió según las sondas empleadas.

\section{Sondas Oncor}

Las láminas fueron deshidratadas a temperatura ambiente en una sucesión de etanol al 70\%, 80\% y 95\% (2 minutos cada). El ADN de los cromosomas fue desnaturalizado en una solución de formamida al $70 \% / 2 X S S C$, (PH 7.0) a $73^{\circ} \mathrm{C}$ durante 2 minutos. Inmediatamente, fueron sometidas a una segunda deshidratación en etanol al $70 \%, 80 \%$ y $95 \%$ a $-20^{\circ} \mathrm{C}$ (2 minutos cada). Paralelamente, las sondas para los cromosomas X y Y (utilizadas simultáneamente) fueron mezcladas según las recomendaciones del fabricante $(1,5 \mu \mathrm{l}$ de la cada sonda y $60 \mu \mathrm{l}$ de Hybrisol VI) y entonces desnaturalizadas a $73^{\circ} \mathrm{C}$ durante 5 minutos. La sonda del cromosoma 21 se encontraba pre-desnaturalizadas y fue simplemente calentada a $37^{\circ} \mathrm{C}$ por 5 minutos. Cerca del área de las láminas fue destinada a la detección de los cromosomas X y Y y la otra mitad al cromosoma 21: $15 \mu \mathrm{l}$ de cada una de las soluciones de sondas fueron colocados sobre cada área destinada, que fue cubierta con laminilla y sellada con "rubber cement". Las preparaciones fueron incubadas durante la noche (12-16 horas) a $37^{\circ} \mathrm{C}$ en cámara húmeda y oscura. Al día siguiente, fueron lavadas 3 veces en una solución de formamida al 50\%/2XSSC 
(5 minutos cada lavado) a $42^{\circ} \mathrm{C}$. Un segundo lavado fue hecho con $2 X S S C$ a $42^{\circ} \mathrm{C}$ y, enseguida, la reacción fue bloqueada con BSA un 3\%/4XSSC/Tween20 un $0,1 \%$, colocándose 20 minutos a $37^{\circ} \mathrm{C}$. La detección fue hecha a través de la adición de $40 \mu \mathrm{l}$ del reactivo adecuado (FITC-avidina/rodamina-antidigoxigenina 1:1 en el caso de los cromosomas X y Y y FITC para el cromosoma 21) por 30 minutos. Tres lavados de 5 minutos cada uno fueron hechos con $\mathrm{PBD}$ a $42^{\circ} \mathrm{C}$. Las láminas fueron coloreadas con DAPI.

\section{Sondas Vysis}

Una vez seleccionadas las áreas de hibridación, se procedió a la desnaturalización del ADN de las preparaciones en una solución de formamida $70 \% / 20 X S S C$ (PH7) a $73^{\circ} \mathrm{C}$ durante 5 minutos. Las preparaciones fueron inmediatamente sometidas a una deshidratación en etanol a temperatura ambiente en concentraciones de 70\%, 85\% y 100\% (un minuto cada pasaje). Después de secas las preparaciones, $10 \mu \mathrm{l}$ de las sondas (pre-desnaturalizadas) fueron aplicadas en las áreas seleccionadas que fueron entonces cubiertas con láminas y selladas con "rubber cement”. Las preparaciones fueron incubadas durante 6 horas, a $37^{\circ} \mathrm{C}$ en cámara húmeda y oscura. Después de ese periodo, fueron lavadas por 2 minutos a $73^{\circ} \mathrm{C}$ en una solución de $0,4 \mathrm{XSSC/} \quad 0,3 \%$ NonidetP 40 . Un segundo lavado fue hecho en solución de $2 \mathrm{XSSC} / \mathrm{un}$ $0,1 \%$ NonidetP40 a temperatura ambiente por un minuto. Después de secas a temperatura ambiente, fueron coloreadas con DAPI II.

\section{Análisis}

El análisis fue realizado con auxilio de un microscopio Zeiss Axioskop, con lámpara de 50W. La captura de las señales fluorescentes fue hecha utilizándose el programa de computador ISIS 3 V3.00 de la Metasystems GmbH. La captura de las señales de los cromosomas X y Y (Oncor) fue hecha con los filtros FITC (490nm) y Rodamina (570nm), y a del cromosoma 21 (Oncor) con el filtro FITC $(490 \mathrm{~nm})$. Para la coloración nuclear fue utilizado el filtro DAPI (360nm). Para análisis de las sondas Vysis, un set de filtros específicos para SpectrumOrange, SpectrumGreen y SpectrumAqua (Vysis) fue utilizado.
De los casos analizados, se contaron aproximadamente 50 núcleos en interface para cada una de las sondas utilizadas. Fueron computados núcleos con uno, dos, tres o más señales. Núcleos interfásicos y cromosomas metafásicos de muestras de líquido amniótico cultivado, con cariotipo conocido, fueron utilizados como control en cada ensayo. Fueron analizados 50 núcleos para evaluar la eficiencia de hibridación, así como la distribución normal de sus señales, para cada una de las sondas utilizadas. Las muestras fueron clasificadas como euploides cuando un 70\% o más de los núcleos presentaban dos señales de hibridación y aneuploides cuando un $70 \%$ o más de los núcleos no presentaban el número de señales esperado para los cromosomas analizados $(5,11)$.

Los resultados de la FISH fueron considerados informativos cuando se observaron núcleos en cantidad suficiente, con señales diferenciadas y de fácil identificación. Núcleos con alta intensidad de marcación de fondo y o/señales yuxtapuestas o poco intensos fueron excluidos del análisis. Señales difusas o irregulares fueron incluidas sólo cuando estaban nítidamente separados. "Split spots" fueron considerados como una única señal si la distancia entre ellos fuera menor que la anchura de uno de ellos; de lo contrario fueron considerados como dos señales. Núcleos con restos de citoplasma o cuyos límites establecieran contacto con otros núcleos fueron excluidos. Muestras con número insuficiente de núcleos, alta intensidad de marcación de fondo y señales de baja intensidad y de difícil identificación fueron consideradas hibridaciones sin éxito. Los resultados fueron comparados con los diagnósticos cromosómicos de la citogenética tradicional.

\section{Resultados}

Fueron analizadas mediante FISH 15 muestras de líquido amniótico no cultivado. La edad gestacional osciló entre $125 / 7$ y 14 6/7 semanas (media \pm 1 d.p. $=13,63 \mathrm{dp}$ 0,52). La edad materna entre $35 \mathrm{y}$ 41 ańos (media \pm 1 d.p. $=38,33 \pm 1,95)$. Todas las pacientes presentaban como principal indicación para la realización del procedimiento la edad materna avanzada (Tabla 1). Cuatro pacientes presentaron, además de la edad materna, otras indicaciones: 
anomalía a la ultrasonografia $(\mathrm{n}=3)$ y anomalía cromosómica en VC (Vellosidad Corial) en mosaico $(\mathrm{n}=1)$. El volumen de líquido amniótico utilizado fue de 2 a $4 \mathrm{ml}$ (media \pm 1 d.p. = 3,16 $\pm 0,58$ ). El tiempo para la obtención de los resultados varió de 1 a 5 días (media \pm 1 d.p. $=2,13 \pm 1,55$ ). El tiempo de entrega de resultados citogenéticos fue de 13 a 22 días $(\mathrm{m} \pm$ 1 d.p. $=18,4 \pm 3,2$ ).

En cada ensayo se determinó la eficiencia de hibridación en núcleos interfásicos de las muestras control. Cuando se utilizaron las sondas Oncor, la media de los núcleos que presentaban dos señales para las sondas analizadas fue del $97,4 \%$, un $95,7 \%$ y un $89,2 \%$ para los cromosomas 21 , XX y XY, respectivamente. En el caso de las sondas Vysis, la media de los núcleos que presentaban dos señales fue del $90,2 \%$, un $83,7 \%$, un $89,8 \%$, un $92,8 \%$, un $94 \%$ para los cromosomas $13,18,21$, XX y XY, respectivamente. Los resultados fueron considerados normales para los cromosomas estudiados cuando las muestras presentaran más del $70 \%$ de los núcleos con las señales de hibridación esperados para una constitución cromosómica normal. En el caso de sondas Oncor, una media del 90,2\% de los núcleos interfásicos presentó señales de hibridación para los dos cromosomas 21, los dos X (o XY). Para las sondas de la Vysis, una media del 87,2\% de los núcleos presentó dos señales para los cromosomas 13, 18, 21 de realización de la amniocentesis. Sin embargo, las señales de hibridación para los tres cromosomas $\mathrm{X}$ fueron identificados en un $84,2 \%$ de los 38 núcleos analizados. En dos casos (14 y 15), no hubo hibridación con las sondas 13/21 de la Vysis, hecho que se repitió en las muestras control y en otros ensayos independientes del presente análisis. Sin embargo, esos mismos casos fueron informativos para las sondas $\mathrm{X} / \mathrm{Y} / 18$. Las cinco aneuploidias diagnosticadas por FISH fueron confirmadas por la citogenética tradicional. Sin embargo, por citogenética se identificó un caso de rearreglo cromosómico equilibrado 46,XY,t(16;17)(q24;q12) pat y un caso de mosaicismo: 46,XX/47,XX,+mar, no diagnosticados por FISH.

En las Tablas 2 y 3 se observan los resultados de hibridación de las muestras que presentaron resultados anormales. Las Figuras 1 y 2 muestran uno de los resultados normales y algunas de las aneuploidias diagnosticadas.

\section{Discusión}

La incorporación de la FISH en el diagnóstico prenatal permitió obtener información sobre las aneuploidias más frecuentes a partir de núcleos interfásicos de células fetales no cultivadas. Con una pequeña muestra de material $(2-5 \mathrm{ml}$ de liquido y X (o X Y).

En la Tabla 1 se observan los resultados obtenidos por FISH y por la citogenética tradicional. Del total, 14 casos $(93,33 \%)$ fueron informativos para los cromosomas estudiados. En la muestra diagnosticada como XXX (caso 2), hubo dificultad en la obtención de 50 núcleos para análisis, probablemente debido a la edad gestacional precoz (12 5/7 semanas)

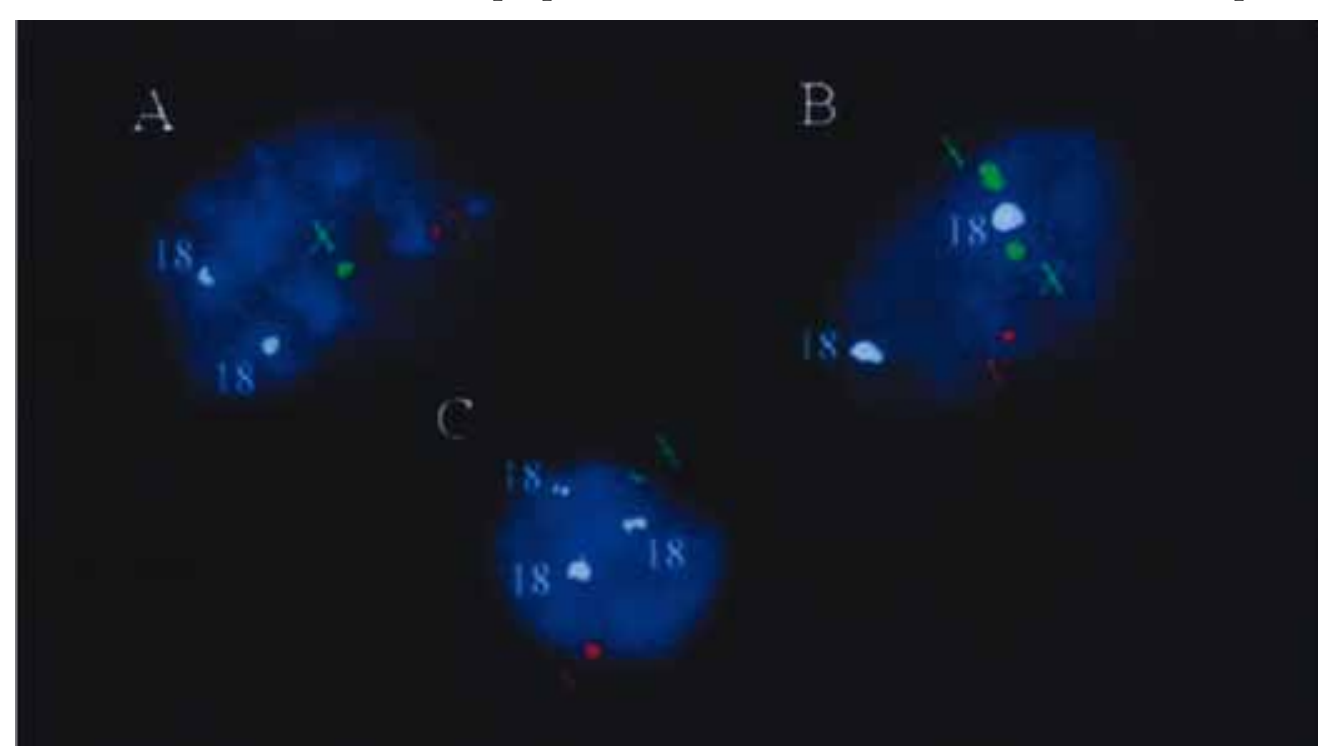

Figura 1. Señales de hibridación en núcleos de amniocitos interfásicos no cultivados. Sonda alfa satélite para los cromosomas X, ZY y 18 (AneuScreen Vysis: DXZ1 SpectrumGreen, DYZ3 SpectrumOrange, D18Z1 SpectrumAqua). A. Núcleo normal para los cromosomas investigados (Xy1818). B. núcleo anormal (XXY1818). C. núcleo anormal (XY181818). 
Tabla 1. Resultados de hibridación in situ por fluorescencia y de la citogenética tradicional.

\begin{tabular}{|c|c|c|c|c|c|c|c|}
\hline Caso & $\begin{array}{c}\text { Edad } \\
\text { materna }\end{array}$ & $\begin{array}{c}\text { Edad } \\
\text { Gestacional }\end{array}$ & Indicación & \multirow{2}{*}{\begin{tabular}{l}
\multicolumn{1}{c}{ Sonda } \\
$\mathrm{X}+\mathrm{Y} / 21$ \\
Oncor
\end{tabular}} & \multicolumn{2}{|c|}{$\begin{array}{l}\text { Resultado } \\
\text { FISH }\end{array}$} & \multirow{2}{*}{\begin{tabular}{|c|c|}
$\begin{array}{c}\text { Resultado } \\
\text { Citogenético }\end{array}$ \\
$47, \mathrm{XXY}$ \\
\end{tabular}} \\
\hline 1 & 41 & $131 / 7$ & Edad materna & & $\begin{array}{l}2(\mathrm{X}) \\
2(21)\end{array}$ & $1(\mathrm{Y})$ & \\
\hline $2^{(a)}$ & 40 & $125 / 7$ & $\begin{array}{l}\text { Edad materna }+ \text { VC } \\
45, \mathrm{X}, 9 \mathrm{qh}+\end{array}$ & $\begin{array}{l}X+Y / 21 \\
\text { Oncor }\end{array}$ & \multicolumn{2}{|c|}{$3(\mathrm{X}) 2(21)$} & $47, \mathrm{XXX}, 9 \mathrm{qh}+$ \\
\hline 3 & 35 & 13 & Edad Materna & $\begin{array}{l}\mathrm{X}+\mathrm{Y} / 21 \\
\text { Oncor }\end{array}$ & \multicolumn{2}{|c|}{$2(\mathrm{X}) 2(21)$} & $46, \mathrm{XX}$ \\
\hline 4 & 37 & $146 / 7$ & Edad Materna & $\begin{array}{l}\mathrm{X}+\mathrm{Y} / 21 \\
\text { Oncor }\end{array}$ & $\begin{array}{l}1(\mathrm{X}) \\
2(21)\end{array}$ & $1(\mathrm{Y})$ & $46, \mathrm{XY}$ \\
\hline 5 & 39 & $133 / 7$ & Edad Materna & $\begin{array}{l}\mathrm{X}+\mathrm{Y} / 21 \\
\text { Oncor }\end{array}$ & $\begin{array}{l}1(\mathrm{X}) \\
2(21)\end{array}$ & $1(\mathrm{Y})$ & $46, X Y$ \\
\hline 6 & 41 & $132 / 7$ & Edad Materna & $\begin{array}{l}\mathrm{X}+\mathrm{Y} / 21 \\
\text { Oncor }\end{array}$ & $\begin{array}{l}1(\mathrm{X}) \\
2(21)\end{array}$ & $1(\mathrm{Y})$ & $\begin{array}{l}46, X Y, t(16,17) \\
\text { (q24;q12)pat }\end{array}$ \\
\hline 7 & 38 & 14 & Edad Materna & $\begin{array}{l}\mathrm{X}+\mathrm{Y} / 21 \\
\text { Oncor }\end{array}$ & \multicolumn{2}{|c|}{$2(\mathrm{X}) 2(21)$} & $46, X X$ \\
\hline 8 & 37 & 14 & Edad Materna & 21 Oncor & \multicolumn{2}{|l|}{$2(21)$} & $46, X X$ \\
\hline 9 & 36 & $132 / 7$ & $\begin{array}{l}\text { Edad Materna + anomalía } \\
\text { de US* }\end{array}$ & 21 Oncor & \multicolumn{2}{|l|}{$3(21)$} & $47, X X .+21$ \\
\hline 10 & 39 & 14 & Edad Materna & 21 Oncor & \multicolumn{2}{|l|}{$2(21)$} & $46, X X$ \\
\hline 11 & 37 & 14 & $\begin{array}{l}\text { Edad Materna + anomalía } \\
\text { de US* }\end{array}$ & 13/21 Vysis & \multicolumn{2}{|c|}{ 2(13) 2(21) } & $46, \mathrm{XX}$ \\
\hline 12 & 41 & 14 & $\begin{array}{l}\text { Edad Materna + anomalía } \\
\text { de US** }\end{array}$ & $\mathrm{X} / \mathrm{Y} / 18$ Vysis & $\begin{array}{l}1(\mathrm{X}) \\
3(18)\end{array}$ & $1(\mathrm{Y})$ & $47, X Y,+18$ \\
\hline & & & & 13/21 Vysis & \multicolumn{2}{|c|}{$2(13) 2(21)$} & \\
\hline 13 & 37 & $146 / 7$ & Edad Materna & X/Y/18 Vysis & \multicolumn{2}{|c|}{$2(\mathrm{X}) 2(18)$} & $46, \mathrm{XX} / 47, \mathrm{XX}+\mathrm{mar}$ \\
\hline & & & & 13/21 Vysis & \multicolumn{2}{|c|}{$2(13) 2(21)$} & \\
\hline \multirow[t]{2}{*}{14} & 37 & 13 & Edad Materna & $\mathrm{X} / \mathrm{Y} / 18$ Vysis & $\begin{array}{l}2(X) \\
2(18)\end{array}$ & $1(\mathrm{Y})$ & $47, \mathrm{XXY}$ \\
\hline & & & & 13/21 Vysis & \multicolumn{2}{|l|}{$\psi$} & \\
\hline 15 & 40 & 13 & Edad Materna & $\mathrm{X} / \mathrm{Y} / 18$ Vysis & $\begin{array}{l}1(\mathrm{X}) \\
2(18)\end{array}$ & $1(\mathrm{Y})$ & $46, \mathrm{XY}$ \\
\hline & & & & 13/21 Vysis & $\psi$ & & \\
\hline
\end{tabular}

\footnotetext{
*Translucencia nucal aumentada ${ }^{* *}$ Translucencia nucal aumentada, onfalocele y retardo de crecimiento intrauterino

(a) Fueron analizados únicamente 38 núcleos

VC: Muestra de vellosidad corial

$\Psi$ ausencia de hibridación
}

amniótico), la FISH permite detectar rápidamente (entre 24 y 48 horas) las aneuploidias de los cromosomas 13, 18, 21, X y Y $(1,6,14,2,4,15)$. Estas corresponden a un $95 \%$ del total de aneuploidias acompañadas de defectos congénitos (16).

Desde la introducción de la FISH en el diagnóstico prenatal, varios trabajos han investigado su precisión. En 1992 fue publicada la primera estandarización de la técnica, así como los protocolos para procesamiento de las sondas. Ya en aquella época la FISH repuntaba como una técnica eficiente en la detección prenatal de las principales aneuploidias (1). En el análisis de Ward $(5,13)$, los resultados fueron informativos en un $89,5 \%$ de los casos y la tasa de detección de aneuploidia de los cromosomas 13, 18, 21, X y Y fue del $78 \%$. Otros estudios han corroborado 
Tabla 2. Distribución de las señales en casos de trisomía.

\begin{tabular}{|cccccc} 
& $\begin{array}{c}\text { Núcleos } \\
\text { Analizados }\end{array}$ & Una señal & Dos señales & Tres señales & Otras \\
\hline Trisomía 21 & 50 & $3(6 \%)$ & $4(8 \%)$ & $41(82 \%)$ & $2(4 \%)$ \\
Trisomía 18 & 50 & $4(8 \%)$ & $3(6 \%)$ & $42(84 \%)$ & $1(2 \%)$ \\
\hline
\end{tabular}

Tabla 3. Porcentaje de señales para los cromosomas X y Y en hibridación simultánea.

\begin{tabular}{lllllllll} 
& \multicolumn{1}{c}{$\begin{array}{c}\text { Núcleos } \\
\text { Analizados }\end{array}$} & \multicolumn{7}{c}{$\%$ de señales (X/Y) } \\
& & $1 / 0$ & $2 / 0$ & $3 / 0$ & $1 / 1$ & $0 / 1$ & $1 / 2$ & $2 / 1$ \\
$\mathrm{XXY}$ & 50 & - & 8 & - & 4 & 4 & - & 84 \\
$\mathrm{XXY}$ & 50 & - & 6 & - & 4 & - & - & 90 \\
$\mathrm{XXX}$ & 38 & 7,9 & 7,9 & 84.2 & - & - & - & - \\
\hline
\end{tabular}

esos hallazgos (10). La comercialización de sondas diseminó la FISH en el diagnóstico prenatal $(14,7,3,17$, 18, 4, 19). La creciente aplicación de la FISH en las instituciones de diagnóstico prenatal nos motivó a probar la aplicabilidad de la técnica en nuestra institución.

La aplicación y precisión de la FISH depende tanto de la especificidad para la hibridación de las

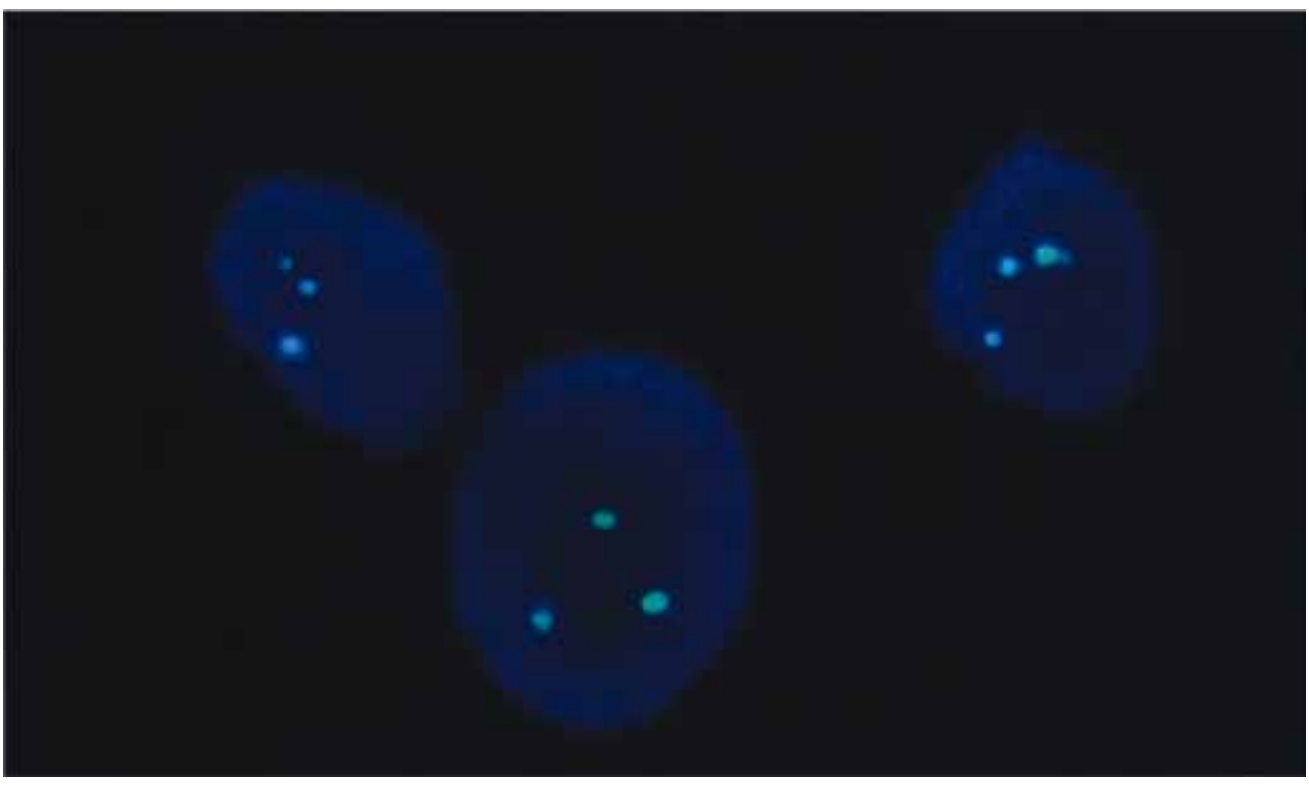

Figura 2. Señales de hibridación en núcleos de amniocitos interfásicos no cultivados. Sonda locus específica para el cromosoma 21 (Oncor -21q22.13-q22.2) Núcleos anorlames con tres señales verdes correspondientes a tres cromosomas 21 .

sondas utilizadas, como

de la estandarización

del protocolo y de la calidad de las muestras (20). En general, las sondas de ADN comercialmente disponibles presentan alta especificidad y los protocolos para a su procesamiento son simple y fáciles de reproducir. La calidad de las muestras es imprescindible. La mayoría de los núcleos interfásicos de las células de líquido amniótico quedan cubiertos por citoplasma, sin revelar señales de hibridación $(21,14)$. El protocolo para la obtención de preparaciones celulares utilizadas en la FISH incluye la lisis de la membrana celular, la eliminación del citoplasma y la fijación adecuada de los núcleos interfásicos. La eficiencia de la FISH y la posibilidad

de obtener núcleos interfásicos con señales de hibridación adecuados depende especialmente de esos factores. Por lo tanto, el protocolo de procesamiento del líquido amniótico es un paso importante, una vez que deben ser obtenidos núcleos en cantidad suficiente para el análisis.

Así como en la mayoría de los trabajos de la literatura, más del $90 \%$ de nuestros resultados fueron informativos. La tasa de detección de aneuploidias fue un $100 \%$. Otros estudios han publicado tasas que varían entre 70 y $100 \%(20,3,2)$. En el estudio de Jalal (2), todos los casos fueron correctamente identificados y no hubo discrepancia con los 
resultados de la citogenética tradicional. Los autores concluyeron que el uso de las sondas comercialmente disponibles podría incrementar la eficiencia de hibridación, además de permitir el análisis de un gran número de células con una pequeña cantidad de LA. Sólo $2 \mathrm{ml}$ de líquido amniótico fueron utilizados en el análisis simultáneo de los cinco cromosomas en 200 núcleos interfásicos.

En nuestra casuística, no fueron observados falsos positivos. Hubo una correlación del $86,7 \%$ entre el resultado de FISH y el resultado de la citogenética tradicional, demostrando alta confiabilidad de la técnica, conforme se indica en otros trabajos (3, 2, 4, 9). La principal desventaja del análisis por FISH en núcleos interfásicos es que solamente pueden ser detectadas las aneuploidias de los cromosomas investigados. Una translocación y un mosaicismo, incluyendo un cromosoma marcador, fueron diagnosticados por citogenética tradicional en la muestra estudiada. En estos casos, la FISH fue normal para los cromosomas investigados.

Solamente en un caso $(6,6 \%)$ no fue posible obtener 50 núcleos para el análisis. Eso puede ser atribuido a la precocidad en que el líquido amniótico fue recolectado. Bryndorf (22) encontraron un 19\% de los casos con menos de 50 núcleos disponibles para análisis. Eiben (3) relataron un $8 \%$ de los casos con menos de 50 núcleos analizados. Las sondas, protocolos utilizados y edad gestacional de realización de la amniocentesis pueden ser los responsables por esa diferencia. Aunque hayamos obtenido sólo 38 núcleos en el caso en cuestión, este nos sugirió anormalidad, pues un alto porcentaje de los núcleos contenía tres señales para el cromosoma $\mathrm{X}$, lo que fue posteriormente confirmado por la citogenética.

Uno de los problemas más frecuentes que presenta el diagnóstico por FISH en líquido amniótico sin cultivar es la presencia de contaminación celular materna. La incidencia de contaminación celular materna en líquido amniótico cultivado es baja y raramente conduce a errores diagnósticos. Pero, la tasa total en muestras no cultivadas puede llegar de más del 20\% (6,23). La forma más visible de contaminación es por la sangre materna (24). Sin embargo, muestras de aspecto macroscópico claro también pueden presentar restos de material materno
(25). Aunque las muestras contaminadas por sangre hayan sido excluidas del análisis y nuestros resultados no hayan sugerido contaminación, no es posible saber si ella estaría presente en muestras con cariótipo femenino normal.

El perfeccionamiento de las técnicas citogenéticas tradicionales ha reducido substancialmente el tiempo medio de entrega de los resultados. Sin embargo, la FISH presenta la gran ventaja de facilitar una información entre 48 y 72 horas. Además de eso, en presencia de un marcador ultrasonográfico sugestivo de aneuploidia, un resultado anormal en FISH puede ser encarado como un diagnóstico definitivo, sin la obligatoriedad de esperar por los resultados del análisis citogenética tradicional.

\section{Agradecimientos}

Este trabajo hace parte de la tesis de doctorado Universidad de Sao Paulo. Beca del "Conselho Nacional de Desenvolvimento Científico e Tecnológico (CNPq)" Brasil.

\section{Referencias}

1. Klinger K., Landes G., Shook D., Harvey R., Lopez L., Locke P., Lerner T., Osathanondh R., Leverone B., Houseal T. (1992). Rapid detection of chromosome aneuploidies in uncultured amniocytes by using flourescence in situ hybridization. Am. J. Hum. Genet., 51:55-65.

2. Jalal S.M., Law M.E., Carlson R.O., Dewald G.W. (1998). Prenatal detection of aneuploidy by directly labeled multicolored probes and interphase fluorescence in situ hybridization.Mayo.Clin. Proc., 73:132-137.

3. Eiben B., Trawicki W., Hammans W., Goebel R., Epplen J.T. (1998). A prospective comparative study on fluorescence in situ hybridization (FISH) of uncultured amniocytes and standard karyotype analysis. Prenat.Diagn., 18:901-906.

4. Morris A., Boyd E., Dhanjal S., Lowther G.W., Aitken D.A., Young J., Menzies A., Imrie S.J., Connor J.M. (1999). Two years prospective experience using flourescence in situ hybridization on uncultured amniotic fluid cells for rapid prenatal diagnosis of common chromosomal aneuploidies. Prenat, Diagn, 19:546-551.

5. WyandtHE,Tonk VS, Huang XL, Evans AT, Milunsky A. Correlation of abnormal rapid FISH and chromosome results from amniocytes for prenatal diagnosis. Fetal DiagnTher 2006; 21: 235-240.

6. Ward B,E., Gersen S.L., Carelli M.P., McGuire N.M., Dackowski W.R., Weinstein M., Sandlin C., Warren R., Klinger K.W. (1993). Rapid prenatal diagnosis of chromosomal aneuploidies by flourescence in situ hybridization: clinical experience with 4500 specimens. Am. J. Hum. Genet, 52:854-865.

7. D’AIton M‘, Malone F.D., Chelmow D., Ward B.E., Bianchi D.W. (1997).Defining the role of fluorescense in situ hybridization on uncultured amniocytes for prenatal diagnosis of aneuploidies. Am. J. Obstet. Gynecol., 176(4):769-776. 
8. Lewin P., Kleinfinger P., Bazin A., Mossafa H., Szpiro-Tapia S. (2000). Defining the efficiency of fluorescence in situ hybridization on uncultured amniocytes on a retrospective cohort of 27407 diagnosis. Prenat.Diagn., 20(1): 1-6.

9. Thilaganathan B., Sairam S., Ballard T., Peterson C., Meredith R. (2000). Effectiveness of prenatal chromosomal analysis using multicolor fluorescet in situ hybridization. Br. J. Obstet. Gynaecol., 107(2):262-266

10. Chan-wei. Fluorescence in situ hybridization in uncultured amniocytesfor detection of aneuploidy in 4210 prenatal cases. JIA Chan-wei, WANG Shu-yu, MA Yan-min, LAN Yong-lian, YU Lan and ZHOU Li-ying. Chin Med J 2011;124(8): 1164-116.

11. American College of Medical Genetics. (1993). Prenatal interphase fluorescence in situ hybrization (FlSH): policy statement. Am. J. Hum. Genet., 53:526-527.

12. Naccache N., Jehee F., Bernal L.M., Gollop T.R. (1996). Amniocentese Precoce entre 12 e 15 semanas de gestação: Relato de 328 casos. Revista da Sociedade Brasileira de Medicina Fetal., 2:35-39.

13. Ward B.E., Gersen S.L., Theve R., Wright M., Carelli M.P. Rapid prenatal diagnosis of cromosomal aneuploidies by flourescence in situ hybridization: clinical experience on 10000 specimens. ln: Pinkel D., Gray J.W. (eds). Clinical Applications of Fluorescence In Situ Hybridization, Chichester: John Wiley, in press. apudBryndorf T., Christensen B., Vad M., Pamer J., Brocks V., Philip J. (1997). Prenatal detection of chromosome aneuploidies by fluorescence in situ hybridization: experience with 2000 uncultured amniotic fluid samples in a prospective preclinical trial. Prenat. $D_{i}$ agn., 17(4):333-341.

14. Carrera M., De la Iglesia C., Méndez B., Ribas 1. (1996). Diagnóstico prenatal rápido de las principales aneuploídias fetales mediante hibridacáo in situ fluorescente: validación de la técnica. Prog. Diagn. Prenat., 8(5): 228-236.

15. Thein A.T., Abdel-Fattah S.A., Kyle P.M,,SoothiIlP.W. (2000). An assessment of the use of interphase FlSH with chromosome specific probes as an alternative to cytogenetic in prenatal diagnosis. Prenat. Diagn., 20(4):275-280.
16. Hook E.B., Cross P.K., Schreinemachers D.M. (1983). Chromosomal abnormality rates at amniocentesis and live-bom infants. JAMA., 249:2034-2038.

17. Verlinsky Y., Ginsberg N., Chmura M., White M., Strom C., Kuliev A. (1998). Detection of translocations involving the Y-chromosome in prospective prenatal screening of common chromosomal aneuploidies by FlSH. Prenat.Diagn., 18:390-392.

18. Pettenati M.J., Berry M.N., Hart P.S., Rao P.N., Lantz P., Rosnes J. (1999).Prenatal interphase detection by FISH of a sex chromosome mosaicism when cytogenetics reports a pseudomosaicism.Prenat. Diagn., 19:25-28.

19. Pergament E., Chen PX, Thangavelu M., Fiddler M. (2000). The clinical application of interphase FISH in prenatal diagnosis. Prenat. Diagn', 20(3):215-220.

20. Divane A., Carter N.P., Spathas D.H., Ferguson-Smith M. A. (1944). Rapid prenatal diagnosis of aneuploidy from uncultured amniotic fluid cells using five-colour fluorescence in situ hybridization. Prenat. Diagn., 14:1061-1069.

21. Cacheux V., Tachdjian G., Druart L., Oury J.F., Sérero 8., Blot P., Nessmann C. (1994). Evaluation of X, Y, 18 and 13/21 alpha satellite DNA probes for interphase cytogenetic analysis of uncultured amniocytes by flourescence in situ hybridization. Prenat.Diagn., 14:79-86.

22. Bryndorf T., Christensen B., Vad M., Pamer J., Brocks V., Philip J. (1997). Prenatal detection of chromosome aneuploidies by fluorescence in situ hybridization: experience with 2000 uncultured amniotic fluid samples in a prospective preclinical trial. Prenat.Diagn., 17(4):333-341.

23. Winsor E.J.T', Silver M.P., Theve R., Wright M., Ward B.E. (1996). Maternal cell contamination in uncultured amniotic fluid.Prenat. Diagn., 16:49-54.

24. Benn P.A., Hsu L.Y.F. (1983). Maternal cell contamination of amniotic fluid cell cultures: results of a US nationwide survey. Am. J. Med. Genet., 15:297-305.

25. Cuatrecasas E., Catalá V., Acevedo MIJ., Crespo M., Serés A. (1996). Contaminación en líquido amniótico detectada mediante citogenética e hibridación in situ. Prog.Diagn.Prenat, 8(5):244-251.
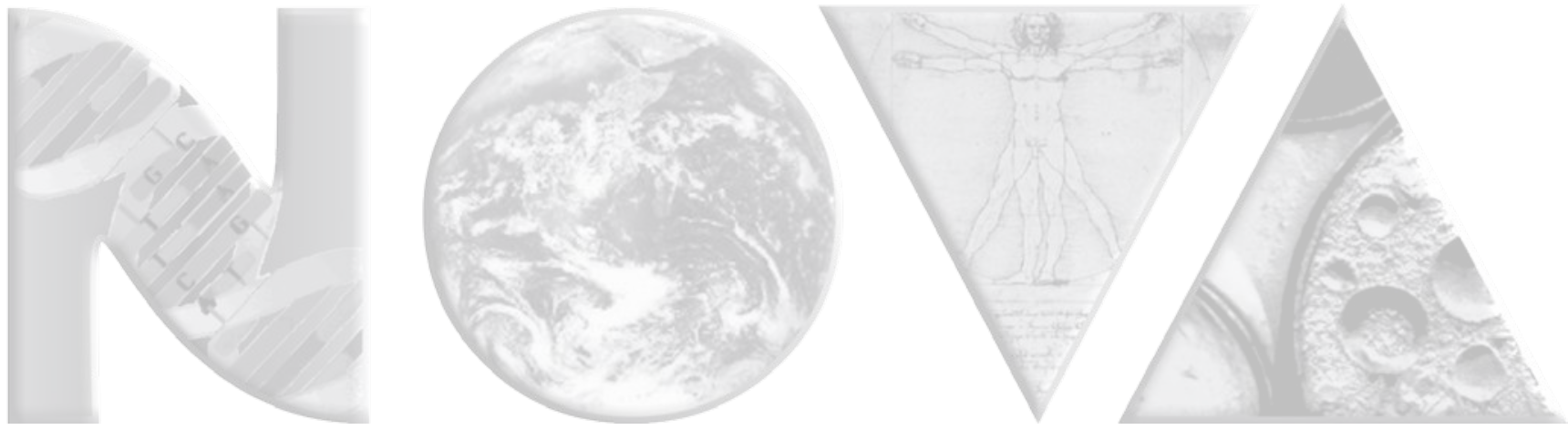